\title{
The high co-existence rate of Blastocystis and Dientamoeba fragilis in human faecal samples and the analysis of demographic and clinical findings
}

\author{
๑Erdoğan Malatyalı, @İbrahim Yıldız, @Evren Tileklioğlu, @Hatice Ertabaklar, @Sema Ertuğ \\ Aydın Adnan Menderes University, Faculty of Medicine, Department of Parasitology, Aydın, Turkey
}

Cite this article as: Malatyalı E, Yıldız İ, Tileklioğlu E, Ertabaklar H, Ertuğ S. The high co-existence rate of Blastocystis and Dientamoeba fragilis in human faecal samples and the analysis of demographic and clinical findings. J Health Sci Med 2021; 4(4): 422-427.

\begin{abstract}
Aim: Blastocystis and Dientamoeba fragilis (D. fragilis) are among the most common protozoon species in human faecal samples. The cross-sectional studies have reported the frequencies in a variety of populations. However, we have very limited information about the co-existence rate of those protozoans. The study aimed to compare D. fragilis frequency in Blastocystis positive and negative faecal samples in order to determine the co-existence rate. The secondary objective was to analyse demographic characteristics and gastrointestinal (GI) symptoms in relation to both infections.

Material and Method: In the present study, we defined a study group that included 100 Blastocystis positive faecal samples and a control group that included 100 Blastocystis negative samples. The frequency of $D$. fragilis in samples was determined with a PCR assay specific to the small-subunit ribosomal RNA (SS rRNA) gene. A positive control of $D$. fragilis was used and the samples with amplification of the expected size ( $863 \mathrm{bp}$ ) were considered as positive. In addition to the statistical comparison of frequencies, the descriptive and clinical findings of cases were analysed retrospectively with Pearson chi-square or ANOVA tests.

Results: The frequency of $D$. fragilis was $21 \%$ in Blastocystis positive group and it was $10 \%$ in Blastocystis negative group. There was statistically significant difference in terms of $D$. fragilis positivity between the groups $(\mathrm{p}<0.05)$. Age, gender and GI symptoms did not reveal a significant difference between the following groups: only Blastocystis infected $(\mathrm{n}=77)$, only $D$. fragilis infected $(\mathrm{n}=11)$, infected with both protozoans ( $\mathrm{n}=34)$ and non-infected individuals $(\mathrm{n}=89)(\mathrm{p}>0.05)$.

Conclusion: Our study highlighted the high co-existence of D. fragilis and Blastocystis in human faecal samples. A possible explanation for this finding may be the faecal-oral transmission of these protozoans. In addition, analysis of clinical findings was supported common asymptomatic colonisation of Blastocystis and D. fragilis.
\end{abstract}

Keywords: Blastocystis, Dientamoeba fragilis, co-existence, clinical findings

\section{INTRODUCTION}

Dientamoeba fragilis ( $D$. fragilis) is a common, globally distributed, enteric protozoon in humans. It was initially classified in amoebas, but the exact taxonomic position was found after phylogenetic and ultra-structural studies. It was finally defined as a member of trichomonads (1). Presence of non-motile pre-cyst and cyst forms in human faecal samples was recently confirmed (2). It colonizes large intestines of humans and some other non-human hosts such as livestock and pet animals. Frequency of $D$. fragilis in many countries has been studied using a variety of diagnostic methods. In common, higher frequencies were reported in developed countries as compared to undeveloped countries unlike other intestinal protozoans (3). Prevalence of $D$. fragilis greatly varied (between $0.4 \%$ and $82.9 \%$ ) in those studies and influenced by the diagnostic methods, study groups, sample size, and geographical location (2). Despite continuous reports emerging over last 100 years, it is still often ignored as a pathogen "neglected parasite", and routine testing often not conducted by diagnostic laboratories. A study from the Netherlands reported that after implementation of faecal PCR, the number of reported $D$. fragilis cases increased 20 folds. In addition, the symptoms in $D$. fragilis infected group lasted longer when compared to Giardia intestinalis infected group and complete resolution of symptoms was noted after eradication of the parasite in faecal samples (4). The previous studies mostly performed in industrialized countries such as the Netherlands and Denmark using molecular techniques and they reported frequencies reaching up to $71 \%$ in particular age groups (5). In Brazil, D. fragilis was detected in $10.3 \%$ of children; the other protozoans were Blastocystis (14.1\%), Endolimax nana 
(13.5\%), Entamoeba coli (12.2\%), and G. intestinalis with a frequency of $10.9 \%$ (6). A study found that frequency of D. fragilis (5.2\%) was lower than Blastocystis (9.6\%) but higher than of G. intestinalis (2\%) among symptomatic and asymptomatic population in Sydney (7). The most common transmission way of intestinal, parasites is faecal-oral and they are mostly defined as food-borne pathogens. World Health Organisation (WHO) reported that the foodborne parasitic diseases including Entamoeba histolytica, G. intestinalis and Cryptosporidium spp. are focal and cause significant morbidity and mortality in vulnerable populations, however, $D$. fragilis was not listed (8).

Previous studies on $D$. fragilis in Turkey reported that the frequencies varied from $0 \%$ to $26.9 \%(9,10)$. However, a systematic and comprehensive understanding of $D$. fragilis prevalence is still lacking in our country. The pathogenic or opportunistic role of $D$. fragilis in human diseases has been a controversial issue for a long period of time. Most of the infected individuals do not represent clinical symptoms and higher frequencies have been reported in healthy group as compared to symptomatic group. Although initially described as a non-pathogen, $D$. fragilis has been associated with wide-ranging symptoms. Symptomatic cases represent primarily non-specific gastrointestinal (GI) symptoms mostly abdominal pain or intermittent diarrhoea (7). In addition, many other symptoms including malaise, nausea, anorexia, fatigue, poor weight gain, and unexplained eosinophilia (almost half of the positive cases) are also attributed to $D$. fragilis infection. The symptoms may persist or re-occur in some patients until application of effective treatment (2).

Blastocystis is an intestinal anaerobic protozoan of humans and many other non-human species. Following the longterm taxonomic studies, Blastocystis was included in the group of Stramenopiles. The colonization in humans and the absence of a flagellated differentiates Blastocystis from others in this group (11). Blastocystis has a global distribution and has been reported as the most common protozoon in human faecal samples in many studies (12). Blastocystis prevalence is higher in undeveloped countries, and frequencies reaching up to $100 \%$ have been reported in Senegal. The evaluation of prevalence studies revealed estimation that 1-2 billion people around the world had Blastocystis infection (13). A study from Sweden evaluated retrospectively the intestinal parasite frequency for 10 -year period, and found that $4.2 \%$ of intestinal parasite prevalence, all of them were positive for Blastocystis. However, it was noted that most had an immigration history (14). Giardia and Blastocystis were the most common protozoan species in a study from Australia (15). A study identified GI pathogens in children with diarrhoea reported $2.9 \%$ Blastocystis carriage and none in asymptomatic group in United Arab Emirates (16). A systematic review in China estimated overall 3.3\% Blastocystis prevalence in the country and noted great variations between cities in terms of Blastocystis frequency, from $0.8 \%$ to $100 \%$ (17). Blastocystis prevalence in Turkey was reported at rates ranging from $1,4 \%$ to $23,5 \%(18,19)$. There is lot of controversy regarding the pathogenicity, genetic diversity, life cycle, diagnosis and treatment of Blastocystis (12). Similar to D. fragilis the role of Blastocystis in the aetiology of particular GI diseases such as irritable bowel syndrome (IBS) and ulcerative colitis (UC) is an important area of interest. Recently, controversial findings suggest that both infections implicate the development of IBS. A systematic review with meta-analysis examined the possible link and reported a correlation with Blastocystis (21). However, there is great need for future studies to reveal the actual mechanisms. Some defined Blastocystis as a pathogen, an opportunistic pathogen, or a non-pathogenic microorganism. Currently, it is thought that Blastocystis pathogenicity is multifactorial and complicated phenomenon that depends on Blastocystis strains, host characteristics, therefore it is hard to explain the pathogenicity over a single feature (12).

Both Blastocystis and D. fragilis are common microorganisms in human faecal samples, worldwide. However, there is limited data about their co-existence and few studies directly investigated this relationship. The aim of the present study was to determine the co-existence of Blastocystis and D. fragilis in human faecal samples with molecular methods. In addition, we aimed to analyse some demographic characteristics and GI symptoms related to Blastocystis and/or D. fragilis infections.

\section{MATERIAL AND METHOD}

The study was reviewed and applied by NoInterventional Clinical Research Ethics Committee of Aydın Adnan Menderes University Faculty of Medicine (Date: 17.02.2021, Decision No: 2021/37). All procedures were carried out in accordance with the ethical rules and the principles of the Declaration of Helsinki.

The study included two groups: Blastocystis positive 100 and Blastocystis negative 100 DNA from faecal samples. All of the 200 individuals were scanned retrospectively for the presence of any other intestinal protozoans or helminths and negatives with direct microscopy were included in the study. Genomic DNAs were previously isolated from faecal samples of the individuals during routine coprological examination in parasitology laboratory in Aydın Adnan Menderes University, Training and Research Hospital. A commercial kit (QIAamp DNA Stool mini kit, Germany) was used to isolate genomic DNA directly from fresh faecal samples. Blastocystis positivity was 
detected by amplification of 18S rRNA gene of Blastocystis with the primers RD5 and BhRDr as previously reported (22). Blastocystis isolates were confirmed by submission of partial 18S rRNA sequences to MLST database (http:// pubmlst.org) and with neighbour-joining method including reference sequences.

\section{Determination of $D$. fragilis Positivity}

Positivity of $D$. fragilis was studied with amplification of the small-subunit rRNA (SSU rRNA) (23). Reaction was set in 30-ul volume: $1 \mu \mathrm{l}$ of template DNA, Taq DNA polymerase $(0.3 \mathrm{U})$, dNTPs $(0.2 \mathrm{mM})$, the primers $(0.4$ pmol), $\mathrm{MgCl}_{2}$ and $1 \times$ Taq buffer with $\left(\mathrm{NH}_{4}\right) 2 \mathrm{SO}_{4}$. The primers DF400 (TAT CGG AGG TGG TAA TGA CC) and DF1250 (CAT CTT CCT CCT GCT TAG ACG) were used in the assay. PCR cycle was as follows: initial denaturation at $94^{\circ} \mathrm{C}$ for $3 \mathrm{~min}, 30$ cycles $\left(1 \mathrm{~min}\right.$ at $94^{\circ} \mathrm{C}, 1.5 \mathrm{~min}$ at $57^{\circ} \mathrm{C}, 2 \mathrm{~min}$ at $72^{\circ} \mathrm{C}$ ) and final extension at $72^{\circ} \mathrm{C}$ for $7 \mathrm{~min}$. The PCR amplicons analysed by electrophoresis on $1 \%$ agarose gel and visualized with the UV imaging system (Vilber Lourmat, France). A previous D. fragilis isolate (ADUDf101), confirmed with partial sequence of SSU rDNA, was used as positive control in our experiment.

\section{Statistical Analysis}

The descriptive data was presented using Statistical Package for the Social Sciences, SPSS (IBM, USA) vs. 21.0. A chisquare test of independence was performed to show the relation between Blastocystis and D. fragilis co-existence rate. The sociodemographic characteristics (gender and age) were evaluated also analysed using Pearson chisquare. The common GI findings were compared between four groups (Blastocystis infected, D. fragilis infected, noninfected and both infected) with ANOVA.

\section{RESULTS}

The positive rate of $D$. fragilis was $23 \%$ in Blastocystis positive samples and it was $11 \%$ in Blastocystis negative samples (Figure, Table 1). D. fragilis positivity was significantly higher in Blastocystis infected individuals as compared to non-infected cases $\chi^{2}(1, \mathrm{~N}=200)=0.1028$, $\mathrm{p}=.0238$. The age of study population $(\mathrm{n}=200)$ varied from 1 to 84 with the average of $37.2 \pm 23.5$. Males accounted for the $\% 53(n=106)$ of individuals and females for $\% 47$ $(n=94)$.

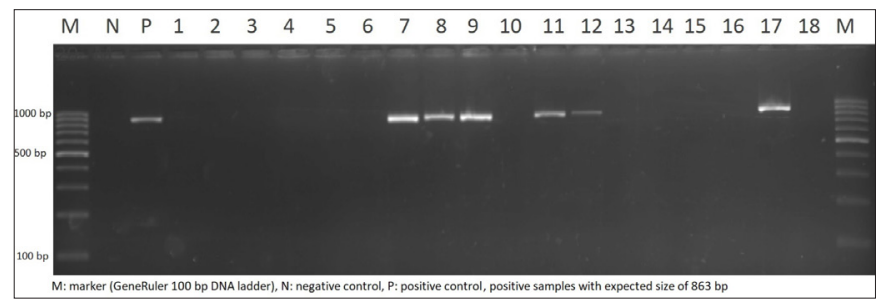

Figure. Agarose gel electrophoresis of amplicons from D. fragilis 18 $S$ rRNA PCR
Table 1. The comparison of D. fragilis frequency in Blastocystis positive and negative samples

\begin{tabular}{|lccc|}
\hline & \multicolumn{3}{c|}{ D. fragilis PCR } \\
\cline { 2 - 4 } & Positive n (\%) & Negative n (\%) & Total \\
\hline Blastocystis & & & \\
Positive & $23(23)$ & $77(77)$ & 100 \\
Negative & $11(11)$ & $89(89)$ & 100 \\
Total & $34(17)$ & $156(78)$ & 200 \\
\hline
\end{tabular}

The faecal samples were sent from many different clinical departments: gastroenterology and hepatology $(\mathrm{n}=53,26.5 \%)$, child health and diseases $(\mathrm{n}=40,20 \%)$, dermatology $(\mathrm{n}=31,15.5 \%)$, general internal medicine $(n=20,10 \%)$, allergy and immunology $(n=17,8.5 \%)$, oncology $(n=10,5 \%)$, infectious diseases $(n=8,4 \%)$, chest diseases $(n=5,2.5 \%)$, and the other departments $(n=16$, $8 \%$ ) including haematology, urology, family medicine, rheumatology, otolaryngology, and nephrology.

The clinical features and diagnosis greatly varied in the study population: abdominal pain $(n=39,19.5 \%)$, diarrhoea $(n=25,12.5 \%)$, allergy $(n=25,12.5 \%)$, flatulence $(\mathrm{n}=24,12 \%)$, constipation $(\mathrm{n}=18,9 \%)$, pruritus $(\mathrm{n}=17,8.5 \%)$, nausea-vomiting $(\mathrm{n}=16,8 \%)$, general medical examination $(\mathrm{n}=14,7 \%)$, malnutritiondevelopmental delay $(n=13,6.5 \%)$, urticeria $(n=12$, $6 \%)$, gastroesophageal reflux $(\mathrm{n}=11,5.5 \%)$, vitamin-D deficiency $(\mathrm{n}=9,4.5 \%)$, anaemia $(\mathrm{n}=7,3.5 \%)$, colitis $(\mathrm{n}=4$, $2 \%)$, dermatitis $(n=4,2 \%)$, dyspepsia $(n=4,2 \%)$, and the others; skin rash, dysuria, cramping, GI haemorrhage, lassitude, myalgia, and cellulitis in single patients. In addition, 12 (6\%) of the studied population were cancer patients, $10(5 \%)$ were ulcerative colitis patients, six (3\%) had irritable bowel syndrome, four had Crohn's disease, four (2\%) had urinary system infections, two (1\%) had pneumonia, two (1\%) had obesity treatment, two (1\%) had renal failure, one had diabetes, and one had rheumatoid arthritis.

In the present study, we found 77 cases with single Blastocystis infection, 11 cases with single $D$. fragilis infection, 34 cases with both of Blastocystis and D. fragilis and 89 non-infected cases. These groups were compared for demographic characteristic in statistical analysis and no significant relation was found regarding gender and age (Table 2). We also analysed common GI findings including abdominal pain, diarrhoea, constipation, flatulence, and nausea-vomiting between the groups, none of these symptoms were significantly different between the groups (Table 3 ). 


\begin{tabular}{|c|c|c|c|c|c|}
\hline & Abdominal pain* & Diarrhoea $^{*}$ & Constipation $^{\star}$ & Flatulence $^{*}$ & Nausea- vomiting $^{*}$ \\
\hline $\begin{array}{l}\text { Blastocystis infected only } \\
(\mathrm{n}=77)\end{array}$ & $15(19.5)$ & $10(12.9)$ & $5(6.4)$ & $8(10.3)$ & $5(6.5)$ \\
\hline $\begin{array}{l}\text { D. fragilis infected only } \\
(\mathrm{n}=11)\end{array}$ & $3(27.3)$ & $2(18.1)$ & $1(9.1)$ & $2(18.1)$ & $1(9.1)$ \\
\hline $\begin{array}{l}\text { Blastocystis and D. fragilis } \\
\text { infected }(\mathrm{n}=34)\end{array}$ & $6(17.6)$ & $4(11.7)$ & $3(8.8)$ & $6(17.6)$ & $2(5.9)$ \\
\hline $\begin{array}{l}\text { Non-infected with both of } \\
\text { them }(n=89)\end{array}$ & $15(16.9)$ & $9(10.1)$ & $9(10.1)$ & $12(13.4)$ & $8(9)$ \\
\hline Chi square (p value) & $0.782(0.852)^{\star \star}$ & $0.775(0.855)^{\star *}$ & $4.873(0.181)^{\star *}$ & $1.354(0.716)^{\star \star}$ & $0.557(0.906)^{\star *}$ \\
\hline
\end{tabular}

Table 2. The comparison of some demographics of studied population between the groups

\begin{tabular}{|c|c|c|c|c|}
\hline & \multirow[b]{2}{*}{$\mathbf{N}$} & \multirow{2}{*}{$\begin{array}{c}\operatorname{Age}^{\star} \\
(\operatorname{mean} \pm s d)\end{array}$} & \multicolumn{2}{|c|}{ Gender $^{\star *}$} \\
\hline & & & $\begin{array}{c}\text { Female } \\
(\mathbf{n}, \%)\end{array}$ & $\begin{array}{c}\text { Male } \\
(\mathbf{n}, \%)\end{array}$ \\
\hline $\begin{array}{l}\text { Blastocystis } \\
\text { infected only }\end{array}$ & 77 & $32.2 \pm 24$ & $37(48.1)$ & 40 (51.9) \\
\hline $\begin{array}{l}\text { D. fragilis infected } \\
\text { only }\end{array}$ & 11 & $42.9 \pm 28.1$ & $5(45.5)$ & $6(54.5)$ \\
\hline $\begin{array}{l}\text { Blastocystis and } \\
\text { D. fragilis infected }\end{array}$ & 34 & $39.6 \pm 22$ & $14(41.2)$ & $20(58.8)$ \\
\hline $\begin{array}{l}\text { Non-infected } \\
\text { with both of them }\end{array}$ & 89 & $36.4 \pm 21.4$ & $38(42.7)$ & $51(57.3)$ \\
\hline
\end{tabular}

\section{DISCUSSION}

In the present study, we have tested the frequency of $D$. fragilis in Blastocystis positive and negative individuals with molecular methods. The findings indicate significantly high infection rate of $D$. fragilis in Blastocystis positive cases. In the literature, previous studies reported some data related to our findings. However, few studies directly aimed to study the co-existence rate with molecular methods. A study investigated the frequency of intestinal parasites in 580 children with diarrhoea and they reported a correlation between Blastocystis and $D$. fragilis. The frequency of $D$. fragilis was $2.7 \%$ among children without Blastocystis infection. However, the rate was $29.6 \%$ among children with Blastocystis infection (24). A study from Iran investigated prevalence of intestinal parasites with conventional parasitological methods and reported that 21 (17\%) of 125 Blastocystis infected individuals were also positive for $D$. fragilis (25). In the Netherlands, $D$. fragilis was detected as the most frequent protozoon in faecal samples of 163 paediatric patients and the combination of D. fragilis and Blastocystis accounted for almost 50\% of them (26). Another study found that Blastocystis frequency was $42 \%$ in D. fragilis positive cases (9 out of 21). D. fragilis was detected as the most common parasite species in faecal samples of studied population (27). Similarly, frequency of $D$. fragilis was studied in patients with GI symptoms and $23.7 \%$ of D. fragilis positives had Blastocystis infection (28). The most common protozoon was $D$. fragilis in Blastocystis infected individuals, 24\% (53 of 221 Blastocystis infected cases). In addition, a significant correlation was found between Blastocystis and D. fragilis (29).

The proposed mode of transmission is faecal-oral for both Blastocystis and D. fragilis $(2,11)$. Therefore, they share a common source of infection for enteric protozoans. Parallel to our findings, a study among IBS patients found that Blastocystis carriage was a risk factor and increased the odds for $D$. fragilis infection (30). In addition, another hypothesis about transmission of $D$. fragilis is the carriage with pinworm eggs (2), our study did not include Enterobius vermicularis positive faecal samples as well as other intestinal parasites. Therefore, we could eliminate possible effects related to this type of transmission in our study.

In our study, the overall positive rate of $D$. fragilis was $17 \%$ in Aydin. In general, the finding was in accordance with the reported frequencies from other cities of Turkey. The frequency of $D$. fragilis was studied in faecal samples collected from 121 individuals, of them 101 had GI complaints and remaining 20 cases were in control group. The overall positive rate of $D$. fragilis was $13 \%$ with iron haematoxylin staining (31). Another study from Istanbul determined $16.7 \%$ positivity of $D$. fragilis and they found a statistically significant difference between healthy individuals and patients in terms of $D$. fragilis positivity (9). In Manisa, $D$. fragilis positivity was studied with different culture methods and $D$. fragilis trophozoites were determined in 11 of $104(10.6 \%)$ samples with Robinson's medium (32). A study from Izmir investigated D. fragilis positivity in 490 faecal samples with real-time PCR; they found that 59 (12\%) patients were infected with $D$. fragilis (28).

A limitation of our study was the possible cross-reaction of PCR testing with other trichomonads in human faecal samples. There is currently no PCR testing protocol for laboratory detection of $D$. fragilis that is approved by U.S. Food and Drug Administration (FDA) and the validation of the tests are still in progress. It was reported that PCR method in our study was tested against various other protozoan parasites including Blastocystis, Entamoeba spp, E. hartmanni, Giardia intestinalis, Endolimax nana, 
Iodamoeba butschlii, Cryptosporidium spp., Cyclospora spp., Chilomastix mesnili, Enteromonas hominis and no amplification was detected with having $100 \%$ specificity (23). However, recently, it was reported that conventional PCR for $D$. fragilis may result in cross reactions with other trichomonads (2). In addition, the sensitivity of PCR testing with DF400 and DF1250 primers was 93.5\% in fresh faecal specimens (23).

Another limitation of the current study was the lack of permanent staining because of the retrospective nature of the study. Faecal samples were initially tested for the presence of Blastocystis and subjected to genomic DNA isolation. Therefore, we could present only wetmount examination (native-Lugol's iodine) of faecal samples from hospital record. At the beginning of the study, we excluded the samples that were positive for other intestinal protozoa. It was reported that the nuclear structure of $D$. fragilis is visible when permanent staining methods are used (2). In general, molecular methods are more sensitive than examination of wetmount preparations. A study reported frequency of $D$. fragilis with direct smear, formalin-ether concentration, culture, permanent staining and amplification of SSU rRNA and 5.8S rRNA genes. The positive rates with the methods were as follows: $0 \%, 0 \%, 1 \%, 5 \%, 6 \%$ and $13.5 \%$, respectively (33).

In the present study, when we compared age, gender and common GI symptoms between the four groups, no statistically significant difference was noted. A number of studies reported no relation between gender and Blastocystis infection, as well as D. fragilis infection, supporting our findings $(33,34)$. A case control study reported that GI symptoms were more common in cases without $D$. fragilis or Blastocystis. In addition, they reported that both $D$. fragilis and Blastocystis frequency was higher in healthy controls than in cases with symptoms (35). However, some reported a correlation with age of cases and Blastocystis infection in particular age groups (19). Despite the relatively small size of study population, the findings on GI can be attributed to the general characteristics of both Blastocystis and D. fragilis infection. Because, there is growing body of literature that reported that these two infections are mostly asymptomatic and a small ratio of infected individuals represent GI symptoms (2,11). Diarrhoea, abdominal pain, constipation and urticarial findings were reported in symptomatic cases of Blastocystis infection (1113). Similar to Blastocystis, non-specific GI symptoms including abdominal pain, cramps and diarrhoea were reported in symptomatic cases of $D$. fragilis (36). Nausea, vomiting, fever and eosinophilia have also been observed in cases with $D$. fragilis infection $(2,37)$. Parallel to these findings, recent developments in the study of microbiota research revealed that the existence of Blastocystis and $D$. fragilis may be related to a healthy intestinal flora $(38,39)$. It was reported that, the colonization of both $D$. fragilis and Blastocystis, unlike bacterial composition, diverged between healthy controls and irritable bowel syndrome (IBS) patients. Their colonization was associated with rich and diverse bacterial microbiota; but the association changed in patients with IBS (40).

\section{CONCLUSION}

The present study reported a significantly high frequency of $D$. fragilis in Blastocystis positive faecal samples. The current finding highlighted the importance of faecaloral transmission of these two protozoa. The analysis of clinical findings emphasises common asymptomatic colonisation of these protozoans. However, the correlation found in our study may not directly indicate a causality and represent a direct relationship of these two pathogens. This finding provides new insights for future research that includes randomized-controlled studies with larger sample size.

\section{ETHICAL DECLARATIONS}

Ethical Committee Approval: The study was reviewed and applied by Non-Interventional Clinical Research Ethics Committee of Aydın Adnan Menderes University Faculty of Medicine (Date: 17.02.2021, Decision No: 2021/37).

Informed Consent: Because of the experimental, retrospective and non-invasive nature of the study design, no written informed consent form was obtained from patients.

Conflict of Interest Statement: The authors declare that they have no conflicts of interest.

Financial Disclosure: No financial support has been received for this study.

Author Contributions: All the authors participated in the design, in the experimental parts, and in the data analysis of the study. All authors approved the final version of the manuscript.

\section{REFERENCES}

1. Silberman JD, Clark CG, Sogin ML. Dientamoeba fragilis shares a recent common evolutionary history with the trichomonads. Mol Biochem Parasitol 1996; 76: 311-4.

2. Garcia LS. Dientamoeba fragilis, one of the neglected intestinal protozoa. J Clin Microbiol 2016; 54: 2243-50.

3. Caccio SM. Molecular epidemiology of Dientamoeba fragilis. Acta Trop 2018; 184: 73-7.

4. Pietila JP, Meri T, Siikamaki H, et al. Dientamoeba fragilis - the most common intestinal protozoan in the Helsinki Metropolitan Area, Finland, 2007 to 2017. European Com Dis Bul 1019; 24: 1800546 
5. Roser D, Simonsen J, Nielsen HV, et al. Dientamoeba fragilis in Denmark: epidemiological experience derived from four years of routine real-time PCR. Eur J Clin Microbiol Infect Dis 2013; 32 1303-10.

6. Oliveira-Arbex AP, David ÉB, Cacciò SM, et al. Prevalence and genetic characterization of Dientamoeba fragilis in asymptomatic children attending daycare centers. Rev Inst Med Trop Sao Paulo 2021; 63, e39.

7. Stark D, Barratt J, Roberts T, Marriott D, Harkness J, Ellis J. A review of the clinical presentation of dientamoebiasis. Am J Trop Med Hyg 2010; 82: 614-9.

8. Torgerson PR, Devleesschauwer B, Praet N, et al. World Health Organization estimates of the global and regional disease burden of 11 foodborne parasitic diseases, 2010: a data synthesis. PLoS Med 2015; 12: e1001920

9. Kurt O, Oktem Okullu S, Ozen NM, et al. Is Dientamoeba fragilis friend or foe? A study from Istanbul on patients and healthy individuals from different cohorts. Turk Mikrobiyol Cem Derg 2020; 50: 244-9.

10. Mumcuoğlu İ, Coşkun FA, Aksu N, Pürnak T, Güngör Ç. İrrite bağırsak sendromunda Dientamoeba fragilis ve Blastocystis spp' nin rolü. Türkiye Parazitol Derg 2013; 37: 73-7.

11. Tan KS. New insights on classification, identification, and clinical relevance of Blastocystis spp. Clin Microbiol Rev 2008; 21: 639-65.

12.Stensvold CR, Clark CG. Current status of Blastocystis: a personal view. Parasitol Int 2016; 65: 763-71.

13. Scanlan PD, Stensvold CR. Blastocystis: getting to grips with our guileful guest. Trends Parasitol 2013; 29: 523-9.

14.Légeret C, Rüttimann C, Fankhauser H, Köhler H. Parasitic infections in Swiss children: are we overtesting? BMC Gastroenterol 2021; 21: 39.

15.Lappan R, Henry R, Chown SL, et al. Monitoring of diverse enteric pathogens across environmental and host reservoirs with TaqMan array cards and standard qPCR: a methodological comparison study. Lancet Planet Health 2021; 5: e297-e308.

16. Alsuwaidi AR, Al Dhaheri K, Al Hamad S, et al. Etiology of diarrhea by multiplex polymerase chain reaction among young children in the United Arab Emirates: a case-control study. BMC Infect Dis 2021; 21: 7.

17.Deng L, Chai Y, Zhou Z, et al. Epidemiology of Blastocystis sp. infection in China: a systematic review. Parasite 2019; 26: 41.

18. Celik T, Daldal N, Karaman U, Aycan MO, Atambay M. Malatya ili merkezinde üç ilköğretim okulu çocuklarında bağırsak parazitlerinin dağılımı. Türkiye Parazitol Derg 2006; 30: 35-8.

19. Hamamci B, Cetinkaya U, Delice S, Erçal BD, Gücüyetmez S, Yazar S. Investigation of intestinal parasites among primary school students in Kayseri-Hacılar. Türkiye Parazitol Derg 2011; 35: 96-9.

20.Mumcuoğlu I, Coşkun FA, Aksu N, Pürnak T, Güngör C. Role of Dientamoeba fragilis and Blastocystis spp. in irritable bowel syndrome. Turkiye Parazitol Derg 2013; 37: 73-7.

21. Rostami A, Riahi SM, Haghighi A, Saber V, Armon B, Seyyedtabaei SJ. The role of Blastocystis sp. and Dientamoeba fragilis in irritable bowel syndrome: a systematic review and meta-analysis. Parasitol Res 2017; 116: 2361-71.

22. Scicluna SM, Tawari B, Clark CG. DNA barcoding of Blastocystis. Protist 2006; 157: 77-85.

23. Stark D, Beebe N, Marriott D, Ellis J, Harkness J. Detection of Dientamoeba fragilis in fresh stool specimens using PCR. Int J Parasitol 2005; 35: 57-62.

24. Boughattas S, Behnke JM, Al-Ansari K, et al. Molecular analysis of the enteric protozoa associated with acute diarrhea in hospitalized children. Front Cell Infect Microbiol 2017; 7: 343.

25.Heydari-Hengami M, Hamedi Y, Najafi-Asl M, Sharifi-Sarasiabi K. Prevalence of intestinal parasites in food handlers of Bandar Abbas, Southern Iran. Iran J Public Health 2018; 47: 111-8.
26. Maas L, Dorigo-Zetsma JW, de Groot CJ, Bouter S, Plötz FB, van Ewijk BE. Detection of intestinal protozoa in paediatric patients with gastrointestinal symptoms by multiplex real-time PCR. Clin Microbiol Infect 2014; 20: 545-50.

27. Norberg A, Nord CE, Evengard B. Dientamoeba fragilis- a protozoal infection which may cause severe bowel distress. Clin Microbiol Infect 2003; 9: 65-8.

28. Aykur M, Caliskan Kurt C, Dirim Erdogan D, et al. Investigation of Dientamoeba fragilis prevalence and evaluation of sociodemographic and clinical features in patients with gastrointestinal symptoms. Acta Parasitol 2019; 64: 162-70.

29. Piubelli C, Soleymanpoor H, Giorli G, et al. Blastocystis prevalence and subtypes in autochthonous and immigrant patients in a referral centre for parasitic infections in Italy. PloS One 2019; 14: e0210171.

30. Engsbro AL, Stensvold CR, Vedel Nielsen H, Bytzer P. Prevalence, incidence, and risk factors of intestinal parasites in Danish primary care patients with irritable bowel syndrome. Scand J Infect Dis 2014; 46: 204-9.

31.Sivcan E, Charyyeva A, Ceylan SS, Yürük M, Erdoğan E, Şahin I. Gastrointestinal şikayeti olan hastalarda Dientamoeba fragilis enfeksiyonu. Mikrobiyol Bul 2018; 52, 166-79.

32. Kurt O, Girginkardesler N, Ozbilgin A, Ok UZ, The effectiveness of the culture for Dientamoeba fragilis from the stool. Infect Dis Clin Microbiol 2019; 1: 34-41.

33. Hamidi N, Meamar AR, Akhlaghi L, Rampisheh Z, Razmjou E. Dientamoeba fragilis diagnosis by fecal screening: relative effectiveness of traditional techniques and molecular methods. J Infect Dev Ctries 2018; 12: 52-9.

34. Dağcı H, Kurt O, Demirel M, et al. Epidemiological and diagnostic features of Blastocystis infection in symptomatic patients in Izmir province, Turkey. Iranian J Parasitol 2014; 9: 519-29.

35. de Boer MD, Schuurs TA, Vermeer M, et al. Distribution and relevance of Dientamoeba fragilis and Blastocystis species in gastroenteritis: results from a case-control study. Eur J Clin Microbiol Infect Dis 2020; 39: 197-203.

36. Hawash YA, Ismail KA, Saber T, et al. Dientamoeba fragilis infection in patients with digestive and non-digestive symptoms: a case-control study. Korean J Parasitol 2020; 58: 129-34.

37. Intra J, Sarto C, Besana S, Tiberti N, Brambilla P. The importance of considering the neglected intestinal protozoan parasite Dientamoeba fragilis. J Med Microbiol 2019; 68: 890-2.

38. O'Brien Andersen L, Karim AB, Roager HM, et al. Associations between common intestinal parasites and bacteria in humans as revealed by qPCR. Eur J Clin Microbiol Infect Dis 2016; 35: 1427 31.

39. Audebert C, Even G, Cian A, et al. Colonization with the enteric protozoa Blastocystis is associated with increased diversity of human gut bacterial microbiota. Sci Rep 2016; 6: 25255.

40. Krogsgaard LR, Andersen LO', Johannesen TB, et al Characteristics of the bacterial microbiome in association with common intestinal parasites in irritable bowel syndrome. Clin Transl Gastroenterol 2018; 9: 161. 